\title{
Maximality Theorems on the Sum of Two Maximal Monotone Operators and Application to Variational Inequality Problems
}

\author{
Teffera M. Asfaw \\ Department of Mathematics, Virginia Polytechnic Institute and State University, Blacksburg, VA 24061, USA \\ Correspondence should be addressed to Teffera M. Asfaw; tefferam@yahoo.com
}

Received 19 May 2016; Accepted 17 July 2016

Academic Editor: Lucas Jodar

Copyright (C) 2016 Teffera M. Asfaw. This is an open access article distributed under the Creative Commons Attribution License, which permits unrestricted use, distribution, and reproduction in any medium, provided the original work is properly cited.

\begin{abstract}
Let $X$ be a real locally uniformly convex reflexive Banach space with locally uniformly convex dual space $X^{*}$. Let $T: X \supseteq D(T) \rightarrow$ $2^{X^{*}}$ and $A: X \supseteq D(A) \rightarrow 2^{X^{*}}$ be maximal monotone operators. The maximality of the sum of two maximal monotone operators has been an open problem for many years. In this paper, new maximality theorems are proved for $T+A$ under weaker sufficient conditions. These theorems improved the well-known maximality results of Rockafellar who used condition $D(T) \cap D(A) \neq \emptyset$ and Browder and Hess who used the quasiboundedness of $T$ and condition $0 \in D(T) \cap D(A)$. In particular, the maximality of $T+\partial \phi$ is proved provided that $D(T) \cap D(\phi) \neq \emptyset$, where $\phi: X \rightarrow(-\infty, \infty]$ is a proper, convex, and lower semicontinuous function. Consequently, an existence theorem is proved addressing solvability of evolution type variational inequality problem for pseudomonotone perturbation of maximal monotone operator.
\end{abstract}

\section{Preliminaries}

In what follows, the norm of spaces $X$ and $X^{*}$ will be denoted by $\|\cdot\|$. For $x \in X$ and $x^{*} \in X^{*}$, pairing $\left\langle x^{*}, x\right\rangle$ denotes value $x^{*}(x)$. Let $X$ and $Y$ be real Banach spaces. For operator $T: X \rightarrow 2^{Y}$, we define domain $D(T)$ of $T$ by $D(T)=\{x \in X:$ $T x \neq \emptyset\}$ and range $R(T)$ of $T$ by $R(T)=\bigcup_{x \in D(T)} T x$. We also use symbol $G(T)$ for the graph of $T: G(T)=\left\{\left(x, x^{*}\right): x \in\right.$ $\left.D(T), x^{*} \in T x\right\}$. A single-valued operator $T: X \supset D(T) \rightarrow$ $Y$ is "demicontinuous," if it is continuous from the strong topology of $D(T)$ to the weak topology of $Y$. It is "compact," if it is strongly continuous and maps bounded subsets of $D(T)$ to relatively compact subsets of $Y$. A multivalued operator $T$ : $X \supset D(T) \rightarrow 2^{Y}$ is "bounded," if it maps each bounded subset of $D(T)$ into a bounded subset of $Y$. It is "finitely continuous," if it is upper semicontinuous from each finite dimensional subspace $F$ of $X$ to the weak topology of $Y$. Throughout the paper, we use notations $z_{n} \rightarrow z_{0}$ and $z_{n} \rightarrow z_{0}$ in $X$ to denote the weak and strong convergence of sequence $\left\{z_{n}\right\}$, respectively. Analogous notations are used for convergence of a sequence in $X^{*}$. Let $\phi:[0, \infty) \rightarrow(-\infty, \infty)$ be a continuous and strictly increasing function such that $\phi(t) \rightarrow \infty$ as $t \rightarrow$ $\infty$. The mapping $J_{\phi}: X \rightarrow 2^{X^{*}}$ defined by

$$
\begin{aligned}
& J_{\phi}(x) \\
& =\left\{x^{*} \in X^{*}:\left\langle x^{*}, x\right\rangle=\phi(\|x\|)\|x\|,\left\|x^{*}\right\|=\phi(\|x\|)\right\}
\end{aligned}
$$

is called the "generalized duality mapping" associated with $\phi$. If $\phi(t)=t$ for all $t \geq 0, J_{\phi}$ is denoted by $J$ and is called "the normalized duality mapping." As a consequence of the Hahn-Banach theorem, it is well-known that $J_{\phi}(x) \neq \emptyset$ for all $x \in X$. Since $X$ and $X^{*}$ are locally uniformly convex, $J_{\phi}$ is single valued, bounded, monotone, and bicontinuous.

Definition 1. An operator $T: X \supset D(T) \rightarrow 2^{X^{*}}$ is said to be

(i) "monotone" if for every $x \in D(T), y \in D(T), u^{*} \in$ $T x$, and $v^{*} \in T y$, one has $\left\langle u^{*}-v^{*}, x-y\right\rangle \geq 0$;

(ii) "maximal monotone" if $T$ is monotone and $R(T+$ $\lambda J)=X^{*}$ for every $\lambda>0$; that is, $T$ is maximal monotone if and only if $T$ is monotone and 
$\left\langle u^{*}-u_{0}^{*}, x-x_{0}\right\rangle \geq 0$ for every $\left(x, u^{*}\right) \in G(T)$ implying $x_{0} \in D(T)$ and $u_{0}^{*} \in T x_{0}$.

The following important lemma is due to Brézis et al. [1].

Lemma 2. Let $B$ be a maximal monotone set in $X \times X^{*}$. If $\left(u_{n}, u_{n}^{*}\right) \in B$ for all $n$ such that $u_{n} \rightarrow u$ in $X$ and $u_{n}^{*} \rightarrow u^{*}$ in $X^{*}$ and either

$$
\limsup _{n, m \rightarrow \infty}\left\langle u_{n}^{*}-u_{m}^{*}, u_{n}-u_{m}\right\rangle \leq 0
$$

or

$$
\limsup _{n \rightarrow \infty}\left\langle u_{n}^{*}-u^{*}, u_{n}-u\right\rangle \leq 0
$$

then $\left(u, u^{*}\right) \in B$ and $\left\langle u_{n}^{*}, u_{n}\right\rangle \rightarrow\left\langle u^{*}, u\right\rangle$ as $n \rightarrow \infty$.

Browder and Hess [2] introduced the following definitions. The original definition of single valued pseudomonotone operator is due to Brézis [3].

Definition 3. An operator $T: X \supset D(T) \rightarrow 2^{X^{*}}$ is said to be "pseudomonotone" if the following conditions are satisfied:

(i) For every $x \in D(T), T x$ is nonempty, closed, convex, and bounded subset of $X^{*}$.

(ii) $T$ is finitely continuous; that is, for every $x_{0} \in D(T) \cap F$ and every weak neighborhood $V$ of $T x_{0}$ in $X^{*}$, there exists neighborhood $U$ of $x_{0}$ in $F$ such that $T U \subset V$.

(iii) For each sequence $\left\{x_{n}\right\} \subset D(T)$ with $y_{n}^{*} \in T x_{n}$ such that $x_{n} \rightarrow x_{0} \in D(T)$ and

$$
\limsup _{n \rightarrow \infty}\left\langle y_{n}^{*}, x_{n}-x_{0}\right\rangle \leq 0
$$

one has that for every $x \in D(T)$, there exists $y^{*}(x) \in$ $T x_{0}$ such that

$$
\left\langle y^{*}(x), x_{0}-x\right\rangle \leq \liminf _{n \rightarrow \infty}\left\langle y_{n}^{*}, x_{n}-x\right\rangle \text {. }
$$

In particular, letting $x_{0}$ in place of $x$ in the above inequality, the pseudomonotonicity of $T$ implies

$$
\liminf _{n \rightarrow \infty}\left\langle y_{n}^{*}, x_{n}-x_{0}\right\rangle \geq 0
$$

For basic properties of monotone type operators, the reader is referred to Browder and Hess [2] and Zeidler [4].

The main contribution of this work is to prove maximality of $T+A$, where $T: X \supseteq D(T) \rightarrow 2^{X^{*}}$ and $A: X \supseteq D(A) \rightarrow$ $2^{X^{*}}$ are maximal monotone operators satisfying only one of the following conditions:

(I) There exist $R>0$ and $x_{0} \in X$ such that, for any bounded subset $B$ of $D(T)$ and each $y \in B\left(x_{0}, R\right)$, there exists number $N(B, y)$ such that

$$
\left\langle g^{*}, x-y\right\rangle \geq N(B, y)
$$

for all $x \in B$ and $g^{*} \in T x$, and for any bounded subset $D$ of $D(A)$, there exists number $N\left(D, x_{0}\right)$ such that

$$
\left\langle w^{*}, x-x_{0}\right\rangle \geq N\left(D, x_{0}\right)
$$

for all $x \in D$ and $w^{*} \in A x$.

(II) $T$ is quasibounded and for a bounded subset $D$ of $D(A)$ there exists number $N(D)$ such that

$$
\left\langle w^{*}, x\right\rangle \geq N(D)
$$

for all $x \in D$ and $w^{*} \in A x$.

It is not difficult to see that (7) is satisfied if $D(T)$ has nonempty interior and (8) is satisfied by $A=\partial \phi$, where $\phi$ : $X \rightarrow(-\infty, \infty]$ is a proper, convex, and lower semicontinuous function with $D(\phi) \neq \emptyset$ and $x_{0} \in D(A)$. Furthermore, both conditions (7) and (8) are satisfied provided that $D(T) \cap$ $D(\phi) \neq \emptyset$, which is weaker than the well-known maximality condition $D(T) \cap D(\partial \phi) \neq \emptyset$ due to Rockafellar [5]. In addition, condition (9) is satisfied if $A=\partial \phi$ with $\phi(0)<\infty$ and $T$ is quasibounded.

The main result due to Rockafellar [5] assumes condition (III) $D(T) \cap D(A) \neq \emptyset$. It easily follows that (III) implies (I); that is, condition (I) is weaker than condition (III). Indeed, if $x_{0} \in D(T) \cap D(A)$, then there exists $R>0$ such that $B\left(x_{0}, R\right) \subset$ $D(T)$. For any bounded subset $B$ of $D(T), y \in B\left(x_{0}, R\right)$, and $h^{*} \in T y$, the monotonicity of $T$ implies that

$$
\begin{aligned}
\left\langle g^{*}, x-y\right\rangle & =\left\langle g^{*}-h^{*}, x-y\right\rangle+\left\langle h^{*}, x-y\right\rangle \\
& \geq\left\langle h^{*}, x-y\right\rangle \geq-\left\|h^{*}\right\|\|x-y\| \\
& \geq-\left(\rho_{B}+\|y\|\right)|T y|=N(B, y)
\end{aligned}
$$

for all $x \in B$ and $g^{*} \in T x$, where $\rho_{B}$ is an upper bounded for $B$ and $|T y|=\inf \left\{\left\|v^{*}\right\|: v^{*} \in T y\right\}$; that is, (7) holds. Similarly, it is not difficult to see that (III) implies (8). Therefore, Theorem 5 improves the well-known maximality result due to Rockafellar [5]. On the other hand, Theorem 10 improves the maximality result due to Browder and Hess [2] which required $T$ to be quasibounded and $0 \in D(T) \cap D(A)$. Theorem 13 provides a new maximality result for $T+\partial \phi$, where $\phi: X \rightarrow(-\infty, \infty]$ is a proper, densely defined, convex, and lower semicontinuous function satisfying mild condition. As a consequence of this maximality result, an existence theorem for solvability of variational inequality problem involving operators of the type $T+S$ with respect to a closed convex subset $K$ of $X$ and the function $\phi$ is included in Theorem 16, where $S: K \rightarrow 2^{X^{*}}$ is a bounded pseudomonotone operator. These results are new and improve analogous results due to Asfaw and Kartsatos [6, Theorem 2.5, Corollary 2.6, Theorem 2.7, pp. 182-187].

The following lemma is useful in the squeal.

Lemma 4. Let $A: X \supseteq D(A) \rightarrow 2^{X^{*}}$ be maximal monotone, $\left\{x_{n}\right\}$ be bounded and there exist $u_{0} \in X$ and $\alpha>0$ such that $\lim \sup _{n \rightarrow \infty}\left\langle A_{\varepsilon_{n}} x_{n}, x_{n}-u_{0}\right\rangle \leq \alpha$, where $\varepsilon_{n} \downarrow 0^{+}$and $A_{\varepsilon_{n}}$ are the Yosida approximant of T. Then, $\left\{J_{\varepsilon_{n}}^{A} x_{n}\right\}$ is bounded, where $J_{\varepsilon_{n}}^{A}$ is the Yosida resolvent of $A$. 
Proof. Let $\varepsilon_{n} \downarrow 0^{+}$and $\left\{x_{n}\right\}$ be bounded. Let $A_{\varepsilon_{n}}$ and $J_{\varepsilon_{n}}^{A}$ be the Yosida approximant and resolvent of $A$, respectively. It is well-known that $J_{\varepsilon_{n}}^{A} x_{n} \in D(A), A_{\varepsilon_{n}} x_{n} \in A\left(J_{\varepsilon_{n}}^{A} x_{n}\right)$, and $J_{\varepsilon_{n}}^{A} x_{n}=x_{n}-\varepsilon_{n} J^{-1}\left(A_{\varepsilon_{n}} x_{n}\right)$ for all $n$. Let $u_{0} \in D(A)$. By the monotonicity of $A_{\varepsilon_{n}}$, we see that

$$
\begin{aligned}
\left\langle A_{\varepsilon_{n}}\right. & \left.x_{n}, x_{n}-u_{0}\right\rangle=\left\langle A_{\varepsilon_{n}} x_{n}, x_{n}-J_{\varepsilon_{n}}^{A} x_{n}+J_{\varepsilon_{n}}^{A} x_{n}-u_{0}\right\rangle \\
= & \left\langle A_{\varepsilon_{n}} x_{n}, x_{n}-J_{\varepsilon_{n}}^{A} x_{n}\right\rangle \\
& +\left\langle A_{\varepsilon_{n}} x_{n}-A_{\varepsilon_{n}} u_{0}, J_{\varepsilon_{n}}^{A} x_{n}-u_{0}\right\rangle \\
& +\left\langle A_{\varepsilon_{n}} u_{0}, J_{\varepsilon_{n}}^{A} x_{n}-u_{0}\right\rangle \\
\geq & \left\langle A_{\varepsilon_{n}} x_{n}, \varepsilon_{n} J^{-1}\left(A_{\varepsilon_{n}} x_{n}\right)\right\rangle-\left|A u_{0}\right|\left\|J_{\varepsilon_{n}}^{A} x_{n}-u_{0}\right\| \\
= & \varepsilon_{n}\left\|A_{\varepsilon_{n}} x_{n}\right\|^{2}-\left|A u_{0}\right|\left\|J_{\varepsilon_{n}}^{A} x_{n}-u_{0}\right\| \\
= & \varepsilon_{n}\left\|A_{\varepsilon_{n}} x_{n}\right\|^{2}-\left|A u_{0}\right|\left\|x_{n}-\varepsilon_{n} J^{-1}\left(A_{\varepsilon_{n}} x_{n}\right)-u_{0}\right\| \\
\geq & \varepsilon_{n}\left\|A_{\varepsilon_{n}} x_{n}\right\|^{2}-\left|A u_{0}\right|\left(\left\|x_{n}\right\|+\varepsilon_{n}\left\|A_{\varepsilon_{n}} x_{n}\right\|+\left\|u_{0}\right\|\right)
\end{aligned}
$$

for all $n$; that is,

$$
\begin{aligned}
\varepsilon_{n}\left\|A_{\varepsilon_{n}} x_{n}\right\|^{2} \leq & \left\langle A_{\varepsilon_{n}} x_{n}, x_{n}-u_{0}\right\rangle \\
& +\left|A u_{0}\right|\left(\left\|x_{n}\right\|+\varepsilon_{n}\left\|A_{\varepsilon_{n}} x_{n}\right\|+\left\|u_{0}\right\|\right) \\
\leq & \alpha+\left|A u_{0}\right|\left(\left\|x_{n}\right\|+\varepsilon_{n}\left\|A_{\varepsilon_{n}} x_{n}\right\|+\left\|u_{0}\right\|\right)
\end{aligned}
$$

for all $n$. Since $J_{\varepsilon_{n}}^{A} x_{n}=x_{n}-\varepsilon_{n} J^{-1}\left(A_{\varepsilon_{n}} x_{n}\right)$ for all $n$, it follows that $\left\{J_{\varepsilon_{n}}^{A} x_{n}\right\}$ is bounded if $\left\{A_{\varepsilon_{n}} x_{n}\right\}$ is bounded. Assume that $\left\{A_{\varepsilon_{n}} x_{n}\right\}$ is unbounded; that is, there exists a subsequence, denoted again by $\left\{A_{\varepsilon_{n}} x_{n}\right\}$, such that $\left\|A_{\varepsilon_{n}} x_{n}\right\| \rightarrow \infty$. Dividing (12) by $\left\|A_{\varepsilon_{n}} x_{n}\right\|$ for all large $n$, we see that

$$
\begin{aligned}
\varepsilon_{n}\left\|A_{\varepsilon_{n}} x_{n}\right\| & \leq \frac{\alpha+\left|A u_{0}\right|\left(\left\|x_{n}\right\|+\varepsilon_{n}\left\|A_{\varepsilon_{n}} x_{n}\right\|+\left\|u_{0}\right\|\right)}{\left\|A_{\varepsilon_{n}} x_{n}\right\|} \\
& \longrightarrow 0,
\end{aligned}
$$

that is, $\varepsilon_{n} A_{\varepsilon_{n}} x_{n} \rightarrow 0$. Since $\left\{x_{n}\right\}$ is bounded and $J_{\varepsilon_{n}}^{A} x_{n}=x_{n}-$ $\varepsilon_{n} J^{-1}\left(A_{\varepsilon_{n}} x_{n}\right)$ for all $n$, it follows that $\left\{J_{\varepsilon_{n}}^{A} x_{n}\right\}$ is bounded.

\section{Main Results}

The following theorem is one of the main results of the paper.
Theorem 5. Let $T: X \supseteq D(T) \rightarrow 2^{X^{*}}$ and $A: X \supseteq D(A) \rightarrow$ $2^{X^{*}}$ be maximal monotone operators such that $D(T) \cap D(A) \neq$ $\emptyset$. Let $B$ be a bounded subset of $D(T)$. Suppose the following conditions are satisfied:

(i) There exist $R>0$ and $x_{0} \in X$ such that for any bounded subset $B$ of $D(T)$ and each $y \in B\left(x_{0}, R\right)$, there exists number $N(B, y)$ such that

$$
\left\langle g^{*}, x-y\right\rangle \geq N(B, y)
$$

for all $x \in B$ and $g^{*} \in T x$.

(ii) For any bounded subset $D$ of $D(A)$, there exists number $N\left(D, x_{0}\right)$ such that

$$
\left\langle w^{*}, x-x_{0}\right\rangle \geq N\left(D, x_{0}\right)
$$

for all $x \in D$ and $w^{*} \in A x$.

Then, $T+A$ is maximal monotone.

Proof. Let $A_{\varepsilon}$ be the Yosida approximants of $A$. Since $T+A_{\varepsilon}$ is maximal monotone, operator $T+A_{\varepsilon}+J$ is surjective; that is, for each $f^{*} \in X^{*}$ and $\varepsilon_{n} \downarrow 0^{+}$, there exist $x_{n} \in D(T)$ and $v_{n}^{*} \in T x_{n}$ such that

$$
v_{n}^{*}+A_{\varepsilon_{n}} x_{n}+J x_{n}=f^{*}
$$

for all $n$. Next, we show that $\left\{x_{n}\right\}$ is bounded. To this end, by choosing $u_{0} \in D(T) \cap D(A), f_{0}^{*} \in T u_{0}$ and applying the monotonicity of $T_{\varepsilon_{n}}$ and $A_{\varepsilon_{n}}$ for all $n$, we see that

$$
\begin{aligned}
\left\langle J x_{n}, x_{n}-u_{0}\right\rangle= & \left\langle f^{*}, x_{n}-u_{0}\right\rangle-\left\langle v_{n}^{*}-f_{0}^{*}, x_{n}-u_{0}\right\rangle \\
& -\left\langle f_{0}^{*}, x_{n}-u_{0}\right\rangle \\
& -\left\langle A_{\varepsilon_{n}} x_{n}-A_{\varepsilon_{n}} u_{0}, x_{n}-u_{0}\right\rangle \\
& -\left\langle A_{\varepsilon_{n}} u_{0}, x_{n}-u_{0}\right\rangle \\
\leq & \left(\left\|f^{*}\right\|+\left\|f_{0}^{*}\right\|+\left\|A_{\varepsilon_{n}} u_{0}\right\|\right)\left\|x_{n}-u_{0}\right\| \\
\leq & \left(\left\|f^{*}\right\|+\left\|f_{0}^{*}\right\|+\left|A u_{0}\right|\right)\left\|x_{n}-u_{0}\right\|
\end{aligned}
$$

for all $n$, where $\left|A u_{0}\right|=\inf \left\{\left\|w^{*}\right\|: w^{*} \in A u_{0}\right\}$. This proves the boundedness of $\left\{x_{n}\right\}$. Next, we show that $\left\{A_{\varepsilon_{n}} x_{n}\right\}$ is bounded. Since $J$ is bounded and $\left\{x_{n}\right\}$ is bounded, it follows that $\left\{J x_{n}\right\}$ is bounded. Let $x_{0} \in X$ and $B\left(x_{0}, R\right)$ satisfy conditions (i) and (ii). Then, we obtain

$$
\begin{aligned}
\left\langle A_{\varepsilon_{n}} x_{n}, x_{n}-y\right\rangle= & \left\langle f^{*}, x_{n}-y\right\rangle-\left\langle v_{n}^{*}, x_{n}-y\right\rangle \\
& -\left\langle J x_{n}, x_{n}-y\right\rangle \\
\leq & \left(\left\|f^{*}\right\|+\left\|x_{n}\right\|\right)\left\|x_{n}-y\right\|-N(B, y) \\
\leq & K(y)-N(B, y)=K_{1}(y)
\end{aligned}
$$


for all $n$, where $K(y)$ is an upper bound for $\left\{\left(\left\|f^{*}\right\|+\left\|x_{n}\right\|\right) \| x_{n}-\right.$ $y \|\}$. Let $J_{\varepsilon_{n}}^{A}$ be the Yosida resolvent of $A$. It is well-known that $J_{\varepsilon_{n}}^{A} x_{n}=x_{n}-\varepsilon_{n} J^{-1}\left(A_{\varepsilon_{n}} x_{n}\right), J_{\varepsilon_{n}}^{A} x_{n} \in D(A)$, and $A_{\varepsilon_{n}} x_{n} \in$ $A\left(J_{\varepsilon_{n}}^{A} x_{n}\right)$. In addition, for any $u_{0} \in D(T)$ and $g_{0}^{*} \in T u_{0}$, by using the monotonicity of $T$, boundedness of $\left\{\left\langle g_{0}^{*}+J x_{n}-\right.\right.$ $\left.\left.f^{*}, x_{n}-u_{0}\right\rangle\right\}$, and (16), we see that

$$
\begin{aligned}
& \limsup _{n \rightarrow \infty}\left\langle A_{\varepsilon_{n}} x_{n}, x_{n}-u_{0}\right\rangle \\
& \leq \limsup _{n \rightarrow \infty}\left(-\left\langle v_{n}^{*}-g_{0}^{*}, x_{n}-u_{0}\right\rangle\right) \\
& \quad+\limsup _{n \rightarrow \infty}\left\langle g_{0}^{*}+J x_{n}-f^{*}, x_{n}-u_{0}\right\rangle \\
& =-\liminf _{n \rightarrow \infty}\left\langle v_{n}^{*}-g_{0}^{*}, x_{n}-u_{0}\right\rangle \\
& \quad+\limsup _{n \rightarrow \infty}\left(\left\|g_{0}^{*}+J x_{n}-f^{*}\right\|\left\|x_{n}-u_{0}\right\|\right) \\
& \leq \limsup _{n \rightarrow \infty}\left(\left\|g_{0}^{*}+J x_{n}-f^{*}\right\|\left\|x_{n}-u_{0}\right\|\right) \leq \alpha,
\end{aligned}
$$

where $\alpha$ is an upper bound for $\left\{\left\|g_{0}^{*}+J x_{n}-f^{*}\right\|\left\|x_{n}-u_{0}\right\|\right\}$. By applying Lemma 4 , we conclude that $\left\{J_{\varepsilon_{n}}^{A} x_{n}\right\}$ is bounded. For each $y \in B\left(x_{0}, R\right)$, applying condition (15) and estimate in (18) yields

$$
\begin{aligned}
&\left\langle A_{\varepsilon_{n}} x_{n}, x_{0}-y\right\rangle=\left\langle A_{\varepsilon_{n}} x_{n}, x_{0}-x_{n}+x_{n}-y\right\rangle \\
&=-\left\langle A_{\varepsilon_{n}} x_{n}, x_{n}-x_{0}\right\rangle+\left\langle A_{\varepsilon_{n}} x_{n}, x_{n}-y\right\rangle \\
&=-\left\langle A_{\varepsilon_{n}} x_{n}, x_{n}-J_{\varepsilon_{n}}^{A} x_{n}+J_{\varepsilon_{n}}^{A} x_{n}-x_{0}\right\rangle \\
&+\left\langle A_{\varepsilon_{n}} x_{n}, x_{n}-y\right\rangle \\
&=-\left\langle A_{\varepsilon_{n}} x_{n}, x_{n}-J_{\varepsilon_{n}}^{A} x_{n}\right\rangle-\left\langle A_{\varepsilon_{n}} x_{n}, J_{\varepsilon_{n}}^{A} x_{n}-x_{0}\right\rangle \\
&+\left\langle A_{\varepsilon_{n}} x_{n}, x_{n}-y\right\rangle \\
&=-\left\langle A_{\varepsilon_{n}} x_{n}, \varepsilon_{n} J^{-1}\left(A_{\varepsilon_{n}} x_{n}\right)\right\rangle \\
&-\left\langle A_{\varepsilon_{n}} x_{n}, J_{\varepsilon_{n}}^{A} x_{n}-x_{0}\right\rangle+\left\langle A_{\varepsilon_{n}} x_{n}, x_{n}-y\right\rangle \\
& \leq-\varepsilon_{n}\left\|A_{\varepsilon_{n}} x_{n}\right\|^{2}-N\left(D, x_{0}\right)+\left\langle A_{\varepsilon_{n}} x_{n}, x_{n}-y\right\rangle \\
& \leq-N\left(D, x_{0}\right)+\left\langle A_{\varepsilon_{n}} x_{n}, x_{n}-y\right\rangle \\
& \leq-N\left(D, x_{0}\right)+K_{1}(y)=K_{2}(y)
\end{aligned}
$$

for all $n$, where $D=\left\{J_{\varepsilon_{n}}^{A} x_{n}\right\}$ which is a bounded subset of $D(A)$. Since $y+x_{0} \in B\left(x_{0}, R\right)$ for all $y \in B(0, R)$, replacing $y+x_{0}$ instead of $y$ in the above inequality, we arrive at

$$
\left\langle A_{\varepsilon_{n}} x_{n}, y\right\rangle \leq-K_{2}\left(y+x_{0}\right)
$$

for all $n$. Since $-y \in B(0, r)$ whenever $y \in B(0, r)$, it follows that there exists $K_{3}(y)>0$ such that

$$
\left|\left\langle A_{\varepsilon_{n}} x_{n}, y\right\rangle\right| \leq K_{3}(y)
$$

for all $n$. Therefore, by applying the uniform boundedness theorem, we conclude that $\left\{A_{\varepsilon_{n}} x_{n}\right\}$ is bounded; that is, $\left\{v_{n}^{*}\right\}$ is bounded. Assume without loss of generality that $x_{n} \rightarrow y_{0}$ (i.e., $J_{\varepsilon_{n}}^{A} x_{n} \rightarrow y_{0}$ ), $v_{n}^{*} \rightarrow v_{0}^{*}$, and $A_{\varepsilon_{n}} x_{n} \rightarrow w_{0}^{*}$. On the other hand, we see that

$$
\begin{aligned}
\limsup _{n \rightarrow \infty}\left\langle A_{\varepsilon_{n}} x_{n}, J_{\varepsilon_{n}}^{A} x_{n}-y_{0}\right\rangle \\
=\limsup _{n \rightarrow \infty}\left(\left\langle A_{\varepsilon_{n}} x_{n}, J_{\varepsilon_{n}}^{A} x_{n}-x_{n}+x_{n}-y_{0}\right\rangle\right) \\
\leq \underset{n \rightarrow \infty}{\limsup }\left\langle A_{\varepsilon_{n}} x_{n}, J_{\varepsilon_{n}}^{A} x_{n}-x_{n}\right\rangle \\
\quad+\limsup _{n \rightarrow \infty}\left\langle A_{\varepsilon_{n}} x_{n}, x_{n}-y_{0}\right\rangle \\
=\limsup _{n \rightarrow \infty}\left(-\left\langle A_{\varepsilon_{n}} x_{n}, \varepsilon_{n} J^{-1}\left(A_{\varepsilon_{n}} x_{n}\right)\right\rangle\right) \\
\quad+\limsup _{n \rightarrow \infty}\left\langle A_{\varepsilon_{n}} x_{n}, x_{n}-y_{0}\right\rangle \\
\leq \limsup _{n \rightarrow \infty}\left(-\varepsilon_{n}\left\|A_{\varepsilon_{n}} x_{n}\right\|^{2}\right) \\
\quad+\limsup _{n \rightarrow \infty}\left\langle A_{\varepsilon_{n}} x_{n}, x_{n}-y_{0}\right\rangle \\
\leq \quad \limsup _{n \rightarrow \infty}\left\langle A_{\varepsilon_{n}} x_{n}, x_{n}-y_{0}\right\rangle .
\end{aligned}
$$

By applying (16) along with monotonicity $J$, we obtain

$$
\begin{aligned}
& \left\langle A_{\varepsilon_{n}} x_{n}, x_{n}-y_{0}\right\rangle \\
& =\limsup _{n \rightarrow \infty}\left(-\left\langle v_{n}^{*}+J x_{n}, x_{n}-y_{0}\right\rangle+\left\langle f^{*}, x_{n}-y_{0}\right\rangle\right) \\
& \leq-\liminf _{n \rightarrow \infty}\left\langle v_{n}^{*}, x_{n}-y_{0}\right\rangle-\liminf _{n \rightarrow \infty}\left\langle J x_{n}, x_{n}-y_{0}\right\rangle \\
& \leq-\liminf _{n \rightarrow \infty}\left\langle v_{n}^{*}, x_{n}-y_{0}\right\rangle .
\end{aligned}
$$

Next, we show that

$$
d=\liminf _{n \rightarrow \infty}\left\langle v_{n}^{*}, x_{n}-y_{0}\right\rangle \geq 0 .
$$

To this end, suppose this is false; that is, $d<0$. Then, there exists a subsequence, denoted again by $\left\{\left\langle v_{n}^{*}, x_{n}-y_{0}\right\rangle\right\}$, such that $\left\langle v_{n}^{*}, x_{n}-y_{0}\right\rangle \rightarrow d$. By applying Lemma 2 , we conclude that $y_{0} \in D(T)$ and $\left\langle v_{n}^{*}, x_{n}\right\rangle \rightarrow\left\langle v_{0}^{*}, y_{0}\right\rangle$. However, this is impossible. Consequently, (16) implies

$$
\limsup _{n \rightarrow \infty}\left\langle A_{\varepsilon_{n}} x_{n}, x_{n}-y_{0}\right\rangle \leq 0
$$

that is, (24) implies

$$
\begin{aligned}
& \limsup _{n \rightarrow \infty}\left\langle A_{\varepsilon_{n}} x_{n}, J_{\varepsilon_{n}}^{A} x_{n}-y_{0}\right\rangle \\
& \quad \leq \limsup _{n \rightarrow \infty}\left\langle A_{\varepsilon_{n}} x_{n}, x_{n}-y_{0}\right\rangle \leq 0 .
\end{aligned}
$$

By the maximality of $A$ along with Lemma 2, we conclude that $y_{0} \in D(T), w_{0}^{*} \in A y_{0}$, and $\left\langle A_{\varepsilon_{n}} x_{n}, J_{\varepsilon_{n}}^{A} x_{n}\right\rangle \rightarrow\left\langle w_{0}^{*}, y_{0}\right\rangle$. 
Similarly, from (16), we obtain $\left\langle v_{n}^{*}, x_{n}-y_{0}\right\rangle \rightarrow 0$. However, this is impossible because $d>0$; that is, $d \geq 0$. As a result, we arrive at

$$
\begin{aligned}
& \limsup _{n \rightarrow \infty}\left\langle J x_{n}, x_{n}-y_{0}\right\rangle \\
& \quad=-\liminf _{n \rightarrow \infty}\left\langle v_{n}^{*}+A_{\varepsilon_{n}} x_{n}-f^{*}, x_{n}-y_{0}\right\rangle \leq 0 .
\end{aligned}
$$

Since $J$ bounded demicontinuous of type $\left(S_{+}\right)$, we conclude that $x_{n} \rightarrow y_{0}$ and $J x_{n} \rightarrow J y_{0}$. Finally, letting $n \rightarrow \infty$ in (16), we conclude that $y_{0} \in D(T) \cap D(A)$ such that $v_{0}^{*}+w_{0}^{*}+J y_{0}=$ $f^{*}$. Since $f^{*} \in X^{*}$ is arbitrary, the surjectivity of $T+A+J$ is proved. Therefore, $T+A$ is maximal monotone. The proof is completed.

It is worth mentioning that Theorem 5 improves the result due to Chen et al. [7, Theorem 2.1, p. 25] because $x_{0} \in X$ can be arbitrary instead of $x_{0} \in D(T) \cap D(A)$, and the side condition can be assumed to hold for all $x \in B$, where $B$ is a bounded subset of $D(T)$ instead of assuming to hold for all $x \in D(T)$ and $-L(B)-\gamma(y)$ can be a number $N(B, y)$ instead of using functions $L$ and $\gamma$ from $X$ into $\mathbb{R}$ with bounded $L$ and $L(B)$ is upper bound for $\{L x: x \in B\}$. In addition, Asfaw [8] used the degree theory developed by himself to prove maximality of sum $T+A$, where $T$ is arbitrary and $A$ is densely defined which satisfies $\Gamma_{\phi}^{\beta}$ condition; that is, there exists a continuous strictly increasing function $\phi:[0, \infty) \rightarrow[0, \infty)$ and, for each $y \in X$, there exists a number $\beta(y)$ such that

$$
\left\langle w^{*}, x-y\right\rangle \geq-\phi(\|x\|)\|x\|-\beta(y)
$$

for all $x \in D(A)$ and $w^{*} \in A x$. In addition, Theorem 5 improved the maximality result due to Asfaw [8, Corollary 1, p. 998]. For further results concerning useful homotopy invariance results, existence theorems, and examples of operators of type $\Gamma_{\phi}^{\beta}$, the reader is referred to the paper due to Asfaw [8].

As a result of Theorem 5, the following corollaries hold.

Corollary 6. Let $T: X \supseteq D(T) \rightarrow 2^{X^{*}}$ and $A: X \supseteq D(A) \rightarrow$ $2^{X^{*}}$ be maximal monotone operators such that $D(T) \cap D(A) \neq$ $\emptyset$. Assume further that there exist $x_{0} \in D(A)$ and $R>0$ such that for each $y \in B\left(x_{0}, R\right)$ and bounded subset $B$ of $D(T)$, there exists number $N(B, y)$ such that

$$
\left\langle g^{*}, x-y\right\rangle \geq N(B, y)
$$

for all $x \in B$ and $g^{*} \in T x$. Then, $T+A$ is maximal monotone.

Proof. Suppose $x_{0} \in D(A)$ and $h_{0}^{*} \in T x_{0}$. By the monotonicity of $A$, we see that

$$
\begin{gathered}
\left\langle w^{*}, x-x_{0}\right\rangle=\left\langle w^{*}-h_{0}^{*}, x-x_{0}\right\rangle+\left\langle h_{0}^{*}, x-x_{0}\right\rangle \\
\geq-\left\|h_{0}^{*}\right\|\left\|x-x_{0}\right\|-\left\|h_{0}^{*}\right\|\|x\| \\
-\left\|h_{0}^{*}\right\|\left\|x_{0}\right\|
\end{gathered}
$$

for all $x \in D(A)$ and $w^{*} \in A x$; that is, condition (ii) of Theorem 5 is satisfied. Since (i) of Theorem 5 is assumed, the maximality of $T+A$ follows by Theorem 5 .
Corollary 7. Let $T: X \supseteq D(T) \rightarrow 2^{X^{*}}$ and $A: X \supseteq D(A) \rightarrow$ $2^{X^{*}}$ be maximal monotone operators such that $D(T) \cap D(A) \neq$ $\emptyset$. Let $D$ be a bounded subset of $D(A)$ :

(i) If there exist $x_{0} \in D(T)$ and number $N\left(D, x_{0}\right)$ such that

$$
\left\langle w^{*}, x-x_{0}\right\rangle \geq N\left(D, x_{0}\right)
$$

for all $x \in D$ and $w^{*} \in A x$, then $T+A$ is maximal monotone.

(ii) Let $\phi: X \rightarrow(-\infty, \infty]$ be a proper, convex, and lower semicontinuous function. If $D(T) \cap D(\phi) \neq \emptyset$, then $T+$ $\partial \phi$ is maximal monotone.

Proof. (i) Let $x_{0} \in D(T)$. Then, there exists $R>0$ such that $B\left(x_{0}, R\right) \subseteq D(T)$. For each $y \in B\left(x_{0}, R\right)$ and $v^{*} \in T y$, the monotonicity of $T$ implies

$$
\begin{aligned}
\left\langle g^{*}, x-y\right\rangle & =\left\langle g^{*}-v^{*}, x-y\right\rangle-\left\|v^{*}\right\|\|x-y\| \\
& \geq-\left\|v^{*}\right\|\|x\|-\left\|v^{*}\right\|\|y\| \\
& \geq-\left\|v^{*}\right\|(\gamma+\|y\|)=N(B, y)
\end{aligned}
$$

for all $x \in B$ and $g^{*} \in T x$, where $\gamma$ is an upper bound for $B$; that is, condition (14) is satisfied. Since (15) holds by the hypothesis, the maximality of $T+A$ follows Theorem 5 .

(ii) Choose $x_{0} \in D(T) \cap D(\phi)$. By the definition of $\partial \phi$, we see that

$$
\left\langle w^{*}, x-x_{0}\right\rangle \geq \phi(x)-\phi\left(x_{0}\right)
$$

for all $x \in D(\partial \phi)$ and $w^{*} \in \partial \phi(x)$. Since $\phi$ is proper, convex, and lower semicontinuous, there exist $h^{*} \in X^{*}$ and number $\beta$ such that $\phi(x) \geq\left\langle h^{*}, x\right\rangle+\beta$; that is, $\phi(x) \geq-\left\|h^{*}\right\|\|x\|+\beta$ for all $x \in D(\phi)$; that is, we have $\left\langle w^{*}, x-x_{0}\right\rangle \geq-\left\|h^{*}\right\|\|x\|-\phi\left(x_{0}\right)+\beta$ for all $x \in D(\partial \phi)$. Let $D$ be a bounded subset of $D(\partial \phi)$. Then, it follows that

$$
\left\langle w^{*}, x-x_{0}\right\rangle \geq-\left\|h^{*}\right\| d-\phi\left(x_{0}\right)+\beta=N\left(D, x_{0}\right)
$$

for all $x \in D$ and $w^{*} \in \partial \phi(x)$, where $d$ is an upper bound for $D$; that is, (ii) of Theorem 5 is satisfied. Thus, the maximality of $T+\partial \phi$ follows by Theorem 5 .

The following well-known result on maximality of the sum of two maximal monotone operators is due to Rockafellar [5]. The proof follows from the conclusion of Theorem 5, which gives a different proof of the maximality criterion due to Rockafellar [5].

Corollary 8. Let $T: X \supseteq D(T) \rightarrow 2^{X^{*}}$ and $A: X \supseteq D(A) \rightarrow$ $2^{X^{*}}$ be maximal monotone operators. If $D(T) \cap D(A) \neq \emptyset$, then $T+A$ is maximal monotone.

Proof. It is easy to see that condition $D(T) \cap D(A) \neq \emptyset$ implies conditions (i) and (ii) of Theorem 5 . The maximality of $T+A$ follows by the conclusion of Theorem 5 . 
Corollary 9. Let $T: X \supseteq D(T) \rightarrow 2^{X^{*}}$ and $A: X \supseteq D(A) \rightarrow$ $2^{X^{*}}$ be maximal monotone operators with $D(T) \cap D(A) \neq \emptyset$. Let $B$ be a bounded subset of $D(T)$. Assume, further, that for each $y \in X$, there exists number $N(B, y)$ such that

$$
\left\langle g^{*}, x-y\right\rangle \geq N(B, y)
$$

for all $x \in B$ and $g^{*} \in T x$. Then $T+A$ is maximal monotone.

Proof. For each $\varepsilon>0$, it follows that $T+A_{\varepsilon}$ is maximal monotone; that is, $T+A_{\varepsilon}+J$ is surjective. Thus, for each $f^{*} \in X^{*}$ and $\varepsilon_{n} \downarrow 0^{+}$, there exist $x_{n} \in D(T)$ and $v_{n}^{*} \in T x_{n}$ such that $v_{n}^{*}+A_{\varepsilon_{n}} x_{n}+J x_{n}=f^{*}$ for all $n$. The surjectivity of $T+A+J$ follows based on the arguments used in the proof of Theorem 5 by using $X$ in place of $B\left(x_{0}, R\right)$. The details are omitted here.

In a recent paper by Chen et al. [7, Theorem 2.5, p. 27], the solvability of the sum of two maximal monotone operators $T$ and $S$ is given under the assumptions in Corollary 9, where $N(B, y)=L(B)+\gamma(y), y \in X, L(B)$ is an upper bounded for $\{L(x): x \in B\}, L$ and $\gamma$ are functions from $X$ into $\mathbb{R}$ with $L$ to be bounded. However, Corollary 9 proves that the sum $T+S$ in Theorem 2.5 due to Chen et al. [7] is maximal monotone and the conclusion of solvability of operator equation involving $T+S$ follows from results for single maximal monotone operator theory.

The second criterion of maximality for $T+A$ is given below. Here, we require $T$ to be quasibounded instead of assuming side conditions. Theorem 10 improves the wellknown result due to Browder and Hess [2].

Theorem 10. Let $T: X \supseteq D(T) \rightarrow 2^{X^{*}}$ and $A: X \supseteq D(A) \rightarrow$ $2^{X^{*}}$ be maximal monotone operators. If $T$ is quasibounded and for any bounded subset $B$ of $D(A)$, there exists number $N(B)$ such that

$$
\left\langle w^{*}, x\right\rangle \geq N(B)
$$

for all $x \in B$ and $w^{*} \in A x$, then $T+A$ is maximal monotone.

Proof. For each $\varepsilon>0$, let $A_{\varepsilon}: X \rightarrow X^{*}$ and $J_{\varepsilon}^{A}: X \rightarrow D(A)$ be the Yosida approximant and resolvent of $A$, respectively. It is well-known that $A_{\varepsilon}$ and $J_{\varepsilon}^{A}$ are everywhere defined bounded and continuous such that $A_{\varepsilon}$ is maximal monotone. It is easy to see that for each $\varepsilon>0$, operator $T+A_{\varepsilon}$ is maximal monotone. As a result, for each $\varepsilon>0$ and $h^{*} \in X^{*}$, there exist unique $x_{\varepsilon} \in D(T)$ and $f_{\varepsilon} \in T x_{\varepsilon}$ such that $f_{\varepsilon}+A_{\varepsilon} x_{\varepsilon}+J x_{\varepsilon}=h^{*}$; that is, for each $\varepsilon_{n} \downarrow 0^{+}$, there exist $x_{n} \in D(T)$ and $f_{n}^{*} \in T x_{n}$ such that

$$
f_{n}^{*}+A_{\varepsilon_{n}} x_{n}+J x_{n}=h^{*}
$$

for all $n$. We will show that $\left\{x_{n}\right\}$ is bounded. Choose $u_{0} \in$ $D(T) \cap D(A)$ and $f_{0}^{*} \in T u_{0}$. Next using (38) and monotonicity of $T$ and $A$, we get

$$
\begin{aligned}
\left\langle J x_{n}, x_{n}-u_{0}\right\rangle= & \left\langle h^{*}, x_{n}-u_{0}\right\rangle-\left\langle f_{n}^{*}, x_{n}-u_{0}\right\rangle \\
& -\left\langle A_{\varepsilon_{n}} x_{n}, x_{n}-u_{0}\right\rangle \\
\leq & \left\|h^{*}\right\|\left\|x_{n}-u_{0}\right\|
\end{aligned}
$$

$$
\begin{aligned}
&-\left\langle f_{n}^{*}-f_{0}^{*}, x_{n}-u_{0}\right\rangle \\
&-\left\langle f_{0}^{*}, x_{n}-u_{0}\right\rangle \\
&-\left\langle A_{\varepsilon_{n}} x_{n}-A_{\varepsilon_{n}} u_{0}, x_{n}-u_{0}\right\rangle \\
&-\left\langle A_{\varepsilon_{n}} u_{0}, x_{n}-u_{0}\right\rangle \\
& \leq\left\|h^{*}\right\|\left\|x_{n}-u_{0}\right\|+\left\|f_{0}^{*}\right\|\left\|x_{n}-u_{0}\right\| \\
&+\left\|A_{\varepsilon_{n}} u_{0}\right\|\left\|x_{n}-u_{0}\right\| \\
& \leq\left\|h^{*}\right\|\left\|x_{n}-u_{0}\right\|+\left\|f_{0}^{*}\right\|\left\|x_{n}-u_{0}\right\| \\
&+\left|A u_{0}\right|\left\|x_{n}-u_{0}\right\|
\end{aligned}
$$

for all $n$, where $\left|A u_{0}\right|=\inf \left\{\left\|w^{*}\right\|: w^{*} \in A u_{0}\right\}$. This shows the boundedness of $\left\{x_{n}\right\}$; that is, $\left\{J x_{n}\right\}$ is bounded. Next, we show that $\left\{J_{\varepsilon_{n}}^{A} x_{n}\right\}$ is bounded. By the monotonicity of $T$, we see that

$$
\begin{aligned}
\left\langle A_{\varepsilon_{n}} x_{n}, x_{n}-u_{0}\right\rangle= & -\left\langle f_{n}^{*}-f_{0}^{*}+f_{0}^{*}, x_{n}-u_{0}\right\rangle \\
& -\left\langle J x_{n}-f^{*}, x_{n}-u_{0}\right\rangle \\
\leq & -\left\langle f_{0}^{*}, x_{n}-u_{0}\right\rangle \\
& -\left\langle J x_{n}-f^{*}, x_{n}-u_{0}\right\rangle \\
\leq & -\left(\left\|f_{0}^{*}\right\|+\left\|J x_{n}-f^{*}\right\|\right)\left\|x_{n}-u_{0}\right\| \\
\leq & K_{4}
\end{aligned}
$$

for all $n$, where $K_{4}$ is a suitable upper bound. By Lemma 4, we conclude that $\left\{J_{\varepsilon_{n}}^{A} x_{n}\right\}$ is bounded. Next, by the condition on $A$, boundedness of $\left\{x_{n}\right\},\left\{J_{\varepsilon_{n}}^{A} x_{n}\right\}$, and $\left\{J x_{n}\right\}$, we see that

$$
\begin{aligned}
\left\langle f_{n}^{*}, x_{n}\right\rangle= & -\left\langle A_{\varepsilon_{n}} x_{n}, x_{n}-J_{\varepsilon_{n}}^{A} x_{n}+J_{\varepsilon_{n}}^{A} x_{n}\right\rangle \\
& +\left\langle f^{*}-J x_{n}, x_{n}\right\rangle \\
= & -\left\langle A_{\varepsilon_{n}} x_{n}, \varepsilon_{n} J^{-1}\left(A_{\varepsilon_{n}} x_{n}\right)\right\rangle \\
& -\left\langle A_{\varepsilon_{n}} x_{n}, J_{\varepsilon_{n}}^{A} x_{n}\right\rangle+\left\langle f^{*}-J x_{n}, x_{n}\right\rangle \\
\leq & -\left\langle A_{\varepsilon_{n}} x_{n}, J_{\varepsilon_{n}}^{A} x_{n}\right\rangle+\left\|f^{*}-J x_{n}\right\|\left\|x_{n}\right\| \\
\leq & -N(B)+\left\|f^{*}-J x_{n}\right\|\left\|x_{n}\right\| \leq \rho
\end{aligned}
$$

for all $n$, where $B=\left\{J_{\varepsilon_{n}}^{A} x_{n}\right\}$ is bounded subset of $D(A), N(B)$ is a number corresponding to $B$ in the hypothesis and $\rho$ is an appropriate upper bound. Since $T$ is quasibounded and $\left\{x_{n}\right\}$ is bounded, we conclude that $\left\{f_{n}^{*}\right\}$ is bounded. Consequently, we arrive at the boundedness of $\left\{A_{\varepsilon_{n}} x_{n}\right\}$. Assume without loss of generality that $x_{n} \rightarrow y_{0}, f_{n}^{*} \rightarrow v_{0}^{*}$, and $A_{\varepsilon_{n}} x_{n} \rightarrow w_{0}^{*}$. Since $T$ is maximal monotone, the argument used in the proof of Theorem 5 along with Lemma 2 gives

$$
\liminf _{n \rightarrow \infty}\left\langle f_{n}^{*}, x_{n}-y_{0}\right\rangle \geq 0 .
$$


As a result, (38) implies

$$
\limsup _{n \rightarrow \infty}\left\langle A_{\varepsilon_{n}} x_{n}, x_{n}-y_{0}\right\rangle \leq 0 .
$$

Since $J_{\varepsilon_{n}}^{A} x_{n} \in D(A), J_{\varepsilon_{n}}^{A} x_{n}=x_{n}-\varepsilon_{n} J^{-1}\left(A_{\varepsilon_{n}} x_{n}\right)$, and $A_{\varepsilon_{n}} x_{n} \in$ $A\left(J_{\varepsilon_{n}}^{A} x_{n}\right)$, it follows that

$$
\begin{aligned}
& \limsup _{n \rightarrow \infty}\left\langle A_{\varepsilon_{n}} x_{n}, J_{\varepsilon_{n}}^{A} x_{n}-y_{0}\right\rangle \\
& =\limsup _{n \rightarrow \infty}\left(\left\langle A_{\varepsilon_{n}} x_{n}, J_{\varepsilon_{n}}^{A} x_{n}-x_{n}\right\rangle+\left\langle A_{\varepsilon_{n}} x_{n}, x_{n}-y_{0}\right\rangle\right) \\
& \leq-\liminf _{n \rightarrow \infty} \varepsilon_{n}\left\|A_{\varepsilon_{n}} x_{n}\right\|^{2}+\limsup _{n \rightarrow \infty}\left\langle A_{\varepsilon_{n}} x_{n}, x_{n}-y_{0}\right\rangle \\
& \leq \limsup _{n \rightarrow \infty}\left\langle A_{\varepsilon_{n}} x_{n}, x_{n}-y_{0}\right\rangle \leq 0 .
\end{aligned}
$$

By Lemma 2, we conclude that $y_{0} \in D(A), w_{0}^{*} \in A y_{0}$, and $\left\langle A_{\varepsilon_{n}} x_{n}, J_{\varepsilon_{n}}^{A} x_{n}\right\rangle \rightarrow\left\langle w_{0}^{*}, y_{0}\right\rangle$, that is, $\left\langle A_{\varepsilon_{n}} x_{n}, x_{n}\right\rangle \rightarrow\left\langle w_{0}^{*}, y_{0}\right\rangle$. Consequently, (38) implies

$$
\limsup _{n \rightarrow \infty}\left\langle J x_{n}, x_{n}-y_{0}\right\rangle \leq 0 .
$$

Since $J$ is demicontinuous of type $\left(S_{+}\right)$, we conclude that $x_{n} \rightarrow$ $y_{0}$ (i.e., $J_{\varepsilon_{n}} x_{n} \rightarrow y_{0}$ ) and $J x_{n} \rightarrow J y_{0}$. Consequently, by using the maximality of $A$ and $T$, we conclude that $y_{0} \in D(T) \cap$ $D(A), v_{0}^{*} \in T y_{0}$, and $w_{0}^{*} \in A y_{0}$ such that $h^{*}=v_{0}^{*}+w_{0}^{*}+J y_{0}$. Since $h^{*} \in X^{*}$ is arbitrary, we conclude that $T+A+J$ is surjective; that is, $T+A$ is maximal monotone. The proof is completed.

In addition, Theorem 10 improves maximality result due to Asfaw and Kartsatos [6, Corollary 2.8, p. 187] using quasiboundedness of $T$ instead of strong quasiboundedness of $T$ with $0 \in T(0)$ and weaker side condition on $A$ instead of the one used by the authors. As a consequence of Theorem 10, we get the following corollary.

Corollary 11. Let $T: X \supseteq D(T) \rightarrow 2^{X^{*}}$ and $A: X \supseteq$ $D(A) \rightarrow 2^{X^{*}}$ be maximal monotone operators. Suppose one of the following conditions holds:

(i) $T$ is quasibounded and $0 \in D(A)$.

(ii) $T$ or $A$ is bounded.

Then $T+A$ is maximal monotone.

Proof. (i) By choosing $h_{0}^{*} \in A(0)$, applying the monotonicity of $A$ gives

$$
\left\langle w^{*}, x\right\rangle=\left\langle w^{*}-h_{0}^{*}, x\right\rangle+\left\langle h_{0}^{*}, x\right\rangle \geq-\left\|h_{0}^{*}\right\|\|x\|,
$$

for all $x \in D(A)$ and $w^{*} \in A x$. That is, condition on $A$ in Theorem 10 is satisfied. Therefore, the maximality of $T+A$ follows by applying Theorem 10 .

(ii) If $A$ is bounded, one can apply Lemma 4 to conclude that $\left\{A_{\varepsilon_{n}} x_{n}\right\}$ is bounded. The maximality of $T+A$ follows by following the arguments used in the proof of Theorems 5 and 10 .
Theorem 10 or Corollary 11 improves the following wellknown maximality result due to Browder and Hess [2, Theorem 9, p. 284].

Corollary 12. Let $T: X \supseteq D(T) \rightarrow 2^{X^{*}}$ and $A$ : $X \supseteq D(A) \rightarrow 2^{X^{*}}$ be maximal monotone operators. If $T$ is quasibounded and $0 \in D(T) \cap D(A)$, then $T+A$ is maximal monotone.

Proof. The proof follows as a particular case of Corollary 11.

The following theorem gives a maximality result for perturbed operator $T+\partial \phi$, where $\phi$ satisfies mild conditions.

Theorem 13. Let $T: X \supseteq D(T) \rightarrow 2^{X^{*}}$ be maximal monotone. Let $\phi: X \rightarrow(-\infty, \infty]$ be proper, densely defined, convex, and lower semicontinuous function. Assume, further, that there exists a nondecreasing continuous function $\beta$ : $[0, \infty) \rightarrow[0, \infty)$ satisfying $\phi(z) \leq \beta(\|z\|)$ for all $z \in D(\phi)$. Then $T+\partial \phi$ is maximal monotone. The same conclusion holds if $T$ is quasibounded and $0 \in D(\phi)$.

Proof. Fix $f^{*} \in X^{*}$. Let $A=\partial \phi$. Let $A_{\varepsilon}$ and $J_{\varepsilon}^{A}$ be the Yosida approximant and resolvent of $A$, respectively. For each $\varepsilon>0$, it follows that $A_{\varepsilon}+T$ is maximal monotone; that is, for each $\varepsilon>0, A_{\varepsilon}+T+J$ is surjective. As a result, for each $\varepsilon_{n} \downarrow 0^{+}$, there exist $x_{n} \in D(T)$ and $v_{n}^{*} \in T x_{n}$ such that $v_{n}^{*}+A_{\varepsilon_{n}} x_{n}+J x_{n}=f^{*}$ for all $n$. We will show that $\left\{x_{n}\right\},\left\{v_{n}^{*}\right\}$, and $\left\{A_{\varepsilon_{n}} x_{n}\right\}$ are bounded. The boundedness of $\left\{x_{n}\right\}$ and $\left\{J_{\varepsilon_{n}}^{A} x_{n}\right\}$ follows by using the arguments in the proof of Theorem 5 and applying Lemma 4, respectively. Next, we show that $\left\{v_{n}^{*}\right\}$ is bounded. Fixing $y \in D(\phi)$, using the boundedness of $\left\{J x_{n}\right\}$ and definition of $\partial \phi$, it follows that

$$
\begin{aligned}
\left\langle v_{n}^{*}, x_{n}-y\right\rangle= & -\left\langle A_{\varepsilon_{n}} x_{n}, x_{n}-y\right\rangle+\left\langle f^{*}, x_{n}-y\right\rangle \\
= & -\left\langle A_{\varepsilon_{n}} x_{n}, x_{n}-J_{\varepsilon_{n}}^{A} x_{n}+J_{\varepsilon_{n}}^{A} x_{n}-y\right\rangle \\
& +\left\langle f^{*}, x_{n}-y\right\rangle \\
= & -\left\langle A_{\varepsilon_{n}} x_{n}, \varepsilon_{n} J^{-1}\left(A_{\varepsilon_{n}} x_{n}\right)\right\rangle \\
& -\left\langle A_{\varepsilon_{n}} x_{n}, J_{\varepsilon_{n}}^{A} x_{n}-y\right\rangle+\left\langle f^{*}, x_{n}-y\right\rangle \\
= & -\varepsilon_{n}\left\|A_{\varepsilon_{n}} x_{n}\right\|^{2}-\left\langle A_{\varepsilon_{n}} x_{n}, J_{\varepsilon_{n}}^{A} x_{n}-y\right\rangle \\
& +\left\langle f^{*}, x_{n}-y\right\rangle \\
\leq & -\left\langle A_{\varepsilon_{n}} x_{n}, J_{\varepsilon_{n}} x_{n}-y\right\rangle+\left\|f^{*}\right\|\left\|x_{n}-y\right\| \\
\leq & -\phi\left(J_{\varepsilon_{n}}^{A} x_{n}\right)+\phi(y)+\left\|f^{*}\right\|\left\|x_{n}-y\right\|
\end{aligned}
$$

for all $y \in D(\phi)$ and $n$. Since $\phi$ is convex and lower semicontinuous, there exist $h^{*} \in X^{*}$ and number $\beta$ such that $\phi(x) \geq\left\langle h^{*}, x\right\rangle+\gamma$ for all $x \in X$. As a result of this and the condition on $\phi$, we get

$$
\left\langle v_{n}^{*}, x_{n}-y\right\rangle \leq\left\|h^{*}\right\| \delta-\gamma+\beta(\|y\|)+M
$$


for all $y \in D(\phi)$, where $\delta$ is an upper bound for sequence $\left\{J_{\varepsilon_{n}}^{A} x_{n}\right\}$ and $M$ is an upper bound for $\left\{\left\|f^{*}\right\|\left\|x_{n}-y\right\|\right\}$. Since $D(\phi)$ is dense in $X$ and $\beta$ is continuous, for each $y \in X$ we have

$$
\left\langle v_{n}^{*}, x_{n}-y\right\rangle \leq\left\|h^{*}\right\| \delta-\gamma+\beta(\|y\|)+M
$$

for all $n$; that is, using $x_{n}-y$ and $x_{n}+y$ in place of $y$ simultaneously, we arrive at

$$
\begin{gathered}
-\left(\left\|f^{*}\right\|\|y\|+\beta\left(\left\|x_{n}+y\right\|\right)-\gamma+\left\|h^{*}\right\| \delta\right) \leq\left\langle v_{n}^{*}, y\right\rangle \\
\leq\left\|h^{*}\right\| \delta-\gamma+\beta\left(\left\|x_{n}-y\right\|\right)+\left\|f^{*}\right\|\|y\|
\end{gathered}
$$

for all $n$; that is, we see that for each $y \in X$, sequence $\left\{\left\langle v_{n}^{*}, y\right\rangle\right\}$ is bounded. The boundedness of $\left\{v_{n}^{*}\right\}$ follows by applying the uniform boundedness principle. Consequently, we conclude that $\left\{A_{\varepsilon_{n}} x_{n}\right\}$ is bounded. The proof of the surjectivity of $T+$ $\partial \phi+J$ is established following the arguments used in the proof of Theorems 5 and 10. The details are omitted here.

The following result provides solvability of variational inequality problem $\operatorname{VIP}\left(T+S, \phi, K, f^{*}\right)$, where $K$ is a nonempty, closed, and convex subset of $X$. We will recall the definition of solvability of variational inequality problem as given in the following definition.

Definition 14. Let $K$ be a nonempty, closed, and convex subset of $X$. The variational inequality problem, denoted by $\operatorname{VIP}(T+$ $\left.S, K, \phi, f^{*}\right)$ is said to be "solvable" in $D(T) \cap D(\phi) \cap K$ if there exist $x_{0} \in D(T) \cap D(\phi) \cap K, v_{0}^{*} \in T x_{0}$, and $w_{0}^{*} \in S x_{0}$ such that

$$
\left\langle v_{0}^{*}+w_{0}^{*}-f^{*}, x-x_{0}\right\rangle \geq \phi\left(x_{0}\right)-\phi(x)
$$

for all $x \in K$.

For any nonempty, bounded, convex, and open subset $G$ of $X$, Definition 14 implies that problem $\operatorname{VIP}(T+S, K \cap$ $\left.\bar{G}, \phi, f^{*}\right)$ is not solvable in $D(T) \cap D(\phi) \cap K \cap \partial G$ if there exists $u_{0} \in K \cap \bar{G}$ such that

$$
\left\langle v^{*}+w^{*}-f^{*}, x-u_{0}\right\rangle>\phi\left(u_{0}\right)-\phi(x)
$$

for all $x \in D(T) \cap D(\phi) \cap K \cap \partial G, v^{*} \in T x$, and $w^{*} \in S x$. Since $D(\partial \phi)$ is a dense subset of $D(\phi)$, it is not difficult to see that the solvability of inclusion

$$
\partial \phi(x)+T x+S x \ni f^{*}
$$

in $D(T) \cap D(S) \cap D(\partial \phi) \cap K$ implies the solvability of problem $\operatorname{VIP}\left(T+S, K, \phi, f^{*}\right)$ in $D(T) \cap D(\phi) \cap K$. If $\phi=I_{K}$, we denote problem $\operatorname{VIP}\left(T+S, K, I_{K}, f^{*}\right)$ just by $\operatorname{VIP}\left(T+S, K, f^{*}\right)$.

In what follows, we will use the following useful lemma due to Asfaw and Kartsatos [6, Lemma 2.2]. It worth mentioning here that Lemma 15 is useful because the global variational inequality problem $\operatorname{VIP}\left(T+S, K, \phi, f^{*}\right)$ is solvable based on the solvability of local problem $\operatorname{VIP}(T+S, K \cap$ $\left.\bar{G}, \phi, f^{*}\right)$ in $D(T) \cap K \cap \bar{G}$ provided that it has no solution in $D(T) \cap K \cap \partial G$.
Lemma 15. Let $K$ be a nonempty, closed, and convex subset of $X$ and $\widetilde{A}: X \supseteq D(\widetilde{A}) \rightarrow 2^{X^{*}}$. Let $G$ be a nonempty, open, and convex subset of $X$. Then, problem $\operatorname{VIP}\left(\widetilde{A}, K, \phi, f^{*}\right)$ is solvable in $D(\widetilde{A}) \cap D(\phi) \cap K \cap G$ provided that problem $\operatorname{VIP}(\widetilde{A}, K \cap$ $\left.\bar{G}, \phi, f^{*}\right)$ is solvable in $D(\widetilde{A}) \cap D(\phi) \cap K \cap G$.

Next we prove the following result.

Theorem 16. Let $K$ be a nonempty, closed, and convex subset of $X$. Let $T: X \supseteq D(T) \rightarrow 2^{X^{*}}$ be maximal monotone, $\phi: X \rightarrow(-\infty, \infty]$ be a proper, densely defined, convex, and lower semicontinuous function, and $S: K \rightarrow 2^{X^{*}}$ be bounded pseudomonotone. Assume, further, that there exists nondecreasing continuous function $\beta:[0, \infty) \rightarrow[0, \infty)$ satisfying $\phi(z) \leq \beta(\|z\|)$ for all $z \in D(\phi)$. Let $f^{*} \in X^{*}$. Suppose one of the following conditions holds:

(i) $K$ is bounded.

(ii) $K$ is unbounded and there exists $u_{0} \in D(T) \cap D(\phi) \cap K$ and $R>0$ such that

$$
\left\langle v^{*}+w^{*}-f^{*}, x-u_{0}\right\rangle+\phi(x)>\phi\left(u_{0}\right)
$$

for all $x \in D(T) \cap K \cap \partial B_{R}(0), v^{*} \in T x$, and $w^{*} \in S x$.

Then variational inequality problem $\operatorname{VIP}\left(T+S, K, \phi, f^{*}\right)$ is solvable in $D(T) \cap K \cap D(\partial \phi)$.

Proof. Suppose (i) holds; that is, $K$ is bounded. Let $A=T+$ $\partial \phi$. By Theorem 13, we have the maximality of $A$. To prove that $\operatorname{VIP}\left(T+S, K, \phi, f^{*}\right)$ is solvable, it is sufficient to show that $\operatorname{VIP}\left(\partial \phi+T+S, K, f^{*}\right)$ is solvable. Let $A_{\varepsilon}$ be the Yosida approximant of $A$. Since $K$ is bounded, it is well-known that $A_{\varepsilon}+S$ is surjective bounded pseudomonotone. Thus, for each $\varepsilon_{n} \downarrow 0^{+}$, there exist $x_{n} \in K$ and $w_{n}^{*} \in S x_{n}$ such that $A_{\varepsilon_{n}} x_{n}+$ $w_{n}^{*}=f^{*}$ for all $n$. Since $\left\{x_{n}\right\}$ and $S$ are bounded, it follows that $\left\{A_{\varepsilon_{n}} x_{n}\right\}$ and $\left\{w_{n}^{*}\right\}$ are bounded. Assume without loss of generality that $x_{n} \rightarrow x_{0}, A_{\varepsilon_{n}} x_{n} \rightarrow v_{0}^{*}$, and $w_{n}^{*} \rightarrow w_{0}^{*}$. By following the arguments used in the proofs of Theorems 5-13 along with Lemma 2, it follows that $x_{0} \in D(A), v_{0}^{*} \in A x_{0}$, and $\left\langle A_{\varepsilon_{n}} x_{n}, J_{\varepsilon_{n}}^{A} x_{n}\right\rangle \rightarrow\left\langle v_{0}^{*}, x_{0}\right\rangle$ and

$$
\limsup _{n \rightarrow \infty}\left\langle w_{n}^{*}, x_{n}-x_{0}\right\rangle \leq 0 .
$$

Since $S$ is pseudomonotone, for each $x \in K$, there exists $y^{*}(x) \in S x_{0}$ such that

$$
\begin{aligned}
& \left\langle y^{*}(x), x_{0}-x\right\rangle \leq \liminf _{n \rightarrow \infty}\left\langle w_{n}^{*}, x_{n}-x\right\rangle \\
& =\liminf _{n \rightarrow \infty}\left(-\left\langle A_{\varepsilon_{n}} x_{n}-f^{*}, x_{n}-J_{\varepsilon_{n}}^{A} x_{n}+J_{\varepsilon_{n}}^{A} x_{n}-x\right\rangle\right) \\
& \leq\left\langle v_{0}^{*}-f^{*}, x_{0}-x\right\rangle,
\end{aligned}
$$

that is, we have

$$
\left\langle y^{*}(x)+v_{0}^{*}-f^{*}, x-x_{0}\right\rangle \geq 0 .
$$

By applying the Hahn-Banach separation theorem, there exists $w_{0}^{*} \in S x_{0}$ such that

$$
\left\langle w_{0}^{*}+v_{0}^{*}-f^{*}, x-x_{0}\right\rangle \geq 0 \quad \forall x \in K .
$$


Since $v_{0}^{*}=g_{0}^{*}+u_{0}^{*}$ with $g_{0}^{*} \in T x_{0}$ and $u_{0}^{*} \in \partial \phi\left(x_{0}\right)$, by using the definition of $\partial \phi\left(x_{0}\right)$, we see that

$$
\left\langle g_{0}^{*}+w_{0}^{*}-f^{*}, x-x_{0}\right\rangle \geq \phi\left(x_{0}\right)-\phi(x)
$$

for all $x \in K$, that is, problem $\operatorname{VIP}\left(T+S, K, \phi, f^{*}\right)$ is solvable. This proves (i). Assume (ii) holds. Since $K_{R}=K \cap$ $\bar{B}_{R}(0)$ is closed, convex, and bounded subset of $X$, applying condition (i) using $K_{R}$ in place of $K$, it follows that $\operatorname{VIP}(T+$ $\left.S, K_{R}, \phi, f^{*}\right)$ is solvable in $D(T) \cap D(\partial \phi) \cap K \cap \bar{B}_{R}(0)$. However, second condition (ii) and Definition 14 imply that problem $\operatorname{VIP}\left(T+S, K_{R}, \phi, f^{*}\right)$ is not solvable in $D(T) \cap D(\partial \phi) \cap \partial B_{R}(0)$. Therefore, by Lemma 15 , we conclude that $\operatorname{VIP}\left(T+S, K, \phi, f^{*}\right)$ is solvable in $D(T) \cap K \cap B_{R}(0)$. The proof is completed.

It worth mentioning that Theorem 16 is new result and improves the result due to Asfaw and Kartsatos [6, Theorem 2.5, p. 182] for solvability of problem $\operatorname{VIP}\left(T+S, K, \phi, f^{*}\right)$ with $\phi$ densely defined and omitting the requirements that $0 \in \stackrel{\circ}{K}$ and $T$ is strongly quasibounded with $0 \in T(0)$. This result is useful because in many variational problems the closed convex subset $K$ can have empty interior.

In the following corollary we use a coercivity-type condition involving the operator $T+S$ and the function $\phi$.

Corollary 17. Let $K$ be a nonempty, closed, and convex subset of $X$. Let $T: X \supseteq D(T) \rightarrow 2^{X^{*}}$ be maximal monotone and $S: K \rightarrow 2^{X^{*}}$ be bounded pseudomonotone. Let $\phi$ : $X \rightarrow(-\infty, \infty]$ be a proper, densely defined, and convex lower semicontinuous function. Assume, further, that there exist a nondecreasing continuous function $\beta:[0, \infty) \rightarrow[0, \infty)$ such that $\phi(z) \leq \beta(\|z\|)$ for all $z \in D(\phi)$, and $u_{0} \in D(\phi) \cap K$ such that

$$
\begin{aligned}
& \inf _{v^{*} \in T x, w^{*} \in S x, x \in D(T) \cap K} \frac{\left\langle v^{*}+w^{*}, x-u_{0}\right\rangle+\phi(x)}{\|x\|} \\
& \longrightarrow \infty .
\end{aligned}
$$

Then for every $f^{*} \in X^{*}$, problem $\operatorname{VIP}\left(T+S, K, \phi, f^{*}\right)$ is solvable in $D(T) \cap D(\phi) \cap K$.

Proof. Since $\phi\left(u_{0}\right)<\infty$, for every $f^{*} \in X^{*}$, there exists $R=$ $R\left(f^{*}\right)>0$, which can be chosen so that $u_{0} \in \bar{B}_{R}(0)$, such that

$$
\left\langle v^{*}+w^{*}-f^{*}, x-u_{0}\right\rangle+\phi(x)>\phi\left(u_{0}\right)
$$

for all $x \in D(T) \cap K \cap \partial B_{R}(0)$. The proof follows from Theorem 16.

In conclusion, we have the following corollary.

Corollary 18. Let $K$ be a nonempty, closed, and convex subset of $X$. Let $T: X \supseteq D(T) \rightarrow 2^{X^{*}}$ be maximal monotone and $S: K \rightarrow 2^{X^{*}}$ be bounded pseudomonotone. Let $\phi: X \rightarrow(-\infty, \infty]$ be proper, densely defined, convex, and lower semicontinuous. Assume, further, that there exist nondecreasing continuous function $\beta:[0, \infty) \rightarrow[0, \infty)$ such that $\phi(z) \leq \beta(\|z\|)$ for all $z \in D(\phi)$, and $u_{0} \in D(\phi) \cap K$ such that

$$
\left\langle v^{*}+w^{*}-f^{*}, x-u_{0}\right\rangle>0
$$

for all $x \in D(T) \cap K$ with sufficiently large $\|x\|, v^{*} \in T x$, and $w^{*} \in S x$. If $\phi(x) \rightarrow \infty$ as $\|x\| \rightarrow \infty$, then problem $\operatorname{VIP}(T+$ $\left.S, K, \phi, f^{*}\right)$ is solvable.

Proof. Choose $R>0$ large enough such that $\left\langle v^{*}+w^{*}-f^{*}, x-\right.$ $\left.u_{0}\right\rangle>0$ and $\phi(x)>\phi\left(u_{0}\right)$ for all $x \in D(T) \cap K \cap \partial B_{R}(0)$, $v^{*} \in T x$, and $w^{*} \in S x$; that is,

$$
\left\langle v^{*}+w^{*}-f^{*}, x-u_{0}\right\rangle+\phi(x)>\phi\left(u_{0}\right)
$$

for all $x \in D(T) \cap K \cap \partial B_{R}(0), v^{*} \in T x$, and $w^{*} \in S x$. The conclusion follows by the argument used in Corollary 17.

For recent existence results concerning variational inequality problems involving monotone type operators, the reader is referred to the papers of Carl and Le $[9,10]$, Carl [11], Carl and Motreanu [12], Kenmochi [13-15], Asfaw and Kartsatos [6], and Asfaw [8, 16, 17] and the references therein.

\section{Competing Interests}

The author declares that there are no competing interests regarding the publication of the paper.

\section{Acknowledgments}

The author would like to thank Virginia Tech for funding the article processing charge.

\section{References}

[1] H. Brézis, M. G. Crandall, and A. Pazy, "Perturbations of nonlinear maximal monotone sets in Banach space," Communications on Pure and Applied Mathematics, vol. 23, pp. 123-144, 1970.

[2] F. E. Browder and P. Hess, "Nonlinear mappings of monotone type in Banach spaces," Journal of Functional Analysis, vol. 11, no. 3, pp. 251-294, 1972.

[3] H. Brézis, "Équations et inéquations non linéaires dans les espaces vectoriels en dualité," Université de Grenoble. Annales de l'Institut Fourier, vol. 18, pp. 115-175, 1968.

[4] E. Zeidler, Nonlinear Functional Analysis and Its Applications, Springer, New York, NY, USA, 1990.

[5] R. T. Rockafellar, "On the maximality of sums of nonlinear monotone operators," Transactions of the American Mathematical Society, vol. 149, pp. 75-88, 1970.

[6] T. M. Asfaw and A. G. Kartsatos, "Variational inequalities for perturbations of maximal monotone operators in reflexive Banach spaces," The Tohoku Mathematical Journal. Second Series, vol. 66, no. 2, pp. 171-203, 2014.

[7] Y. Chen, Y. Cho, and P. Kumam, "On the maximality of sums of two maximal monotone operators," Journal of Mathematical Analysis, vol. 7, no. 2, pp. 24-30, 2016. 
[8] T. M. Asfaw, "A new topological degree theory for pseudomonotone perturbations of the sum of two maximal monotone operators and applications," Journal of Mathematical Analysis and Applications, vol. 434, no. 1, pp. 967-1006, 2016.

[9] S. Carl and V. K. Le, "Quasilinear parabolic variational inequalities with multi-valued lower-order terms," Zeitschrift für Angewandte Mathematik und Physik, vol. 65, no. 5, pp. 845-864, 2014.

[10] S. Carl, V. K. Le, and D. Motreanu, "Existence, comparison, and compactness results for quasilinear variational-hemivariational inequalities," International Journal of Mathematics and Mathematical Sciences, no. 3, pp. 401-417, 2005.

[11] S. Carl, "Existence and comparison results for noncoercive and nonmonotone multivalued elliptic problems," Nonlinear Analysis: Theory, Methods \& Applications, vol. 65, no. 8, pp. 1532-1546, 2006.

[12] S. Carl and D. Motreanu, "General comparison principle for quasilinear elliptic inclusions," Nonlinear Analysis: Theory, Methods \& Applications, vol. 70, no. 2, pp. 1105-1112, 2009.

[13] N. Kenmochi, "Nonlinear operators of monotone type in reflexive Banach spaces and nonlinear perturbations," Hiroshima Mathematical Journal, vol. 4, pp. 229-263, 1974.

[14] N. Kenmochi, "Pseudomonotone operators and nonlinear elliptic boundary value problems," Journal of the Mathematical Society of Japan, vol. 27, pp. 121-149, 1975.

[15] N. Kenmochi, "Monotonicity and compactness methods for nonlinear variational inequalities," in Handbook of Differential Equations, IV, pp. 203-298, Elsevier/North-Holland, Amsterdam, The Netherlands, 2007.

[16] T. M. Asfaw, "New surjectivity results for perturbed weakly coercive operators of monotone type in reflexive Banach spaces," Nonlinear Analysis: Theory, Methods \& Applications, vol. 113, pp. 209-229, 2015.

[17] T. M. Asfaw, "New variational inequality and surjectivity theories for perturbed noncoercive operators and application to nonlinear problems," Advances in Mathematical Sciences and Applications, vol. 24, no. 2, pp. 611-668, 2014. 


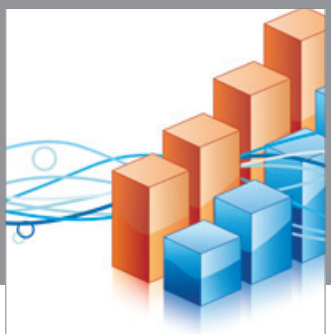

Advances in

Operations Research

vatem alat4

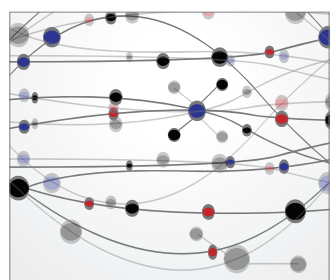

\section{The Scientific} World Journal
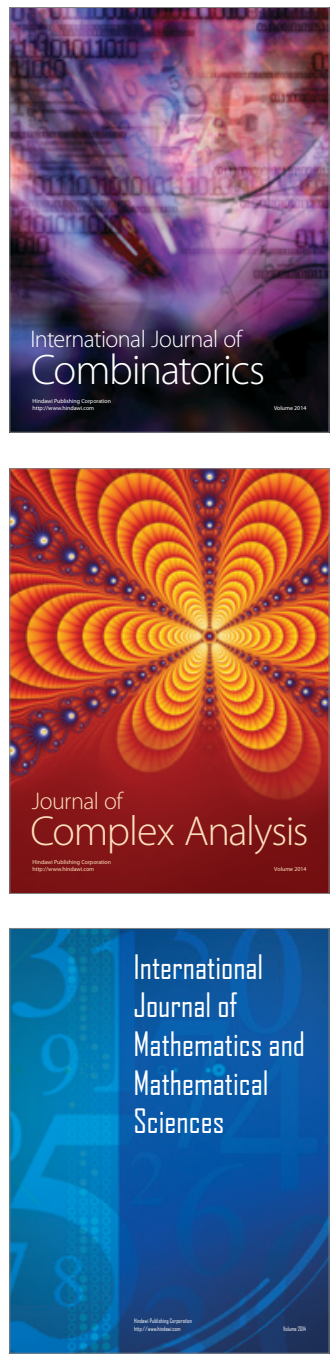
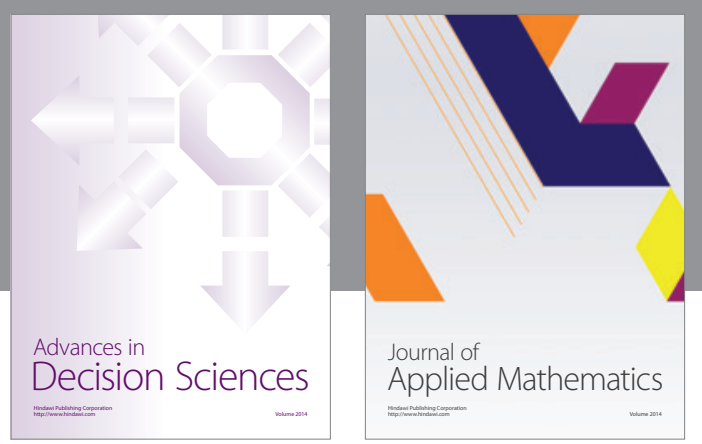

Algebra

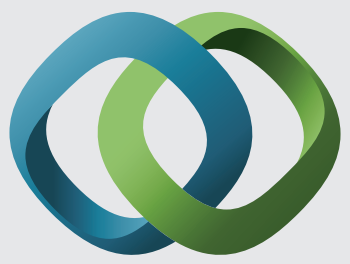

\section{Hindawi}

Submit your manuscripts at

http://www.hindawi.com
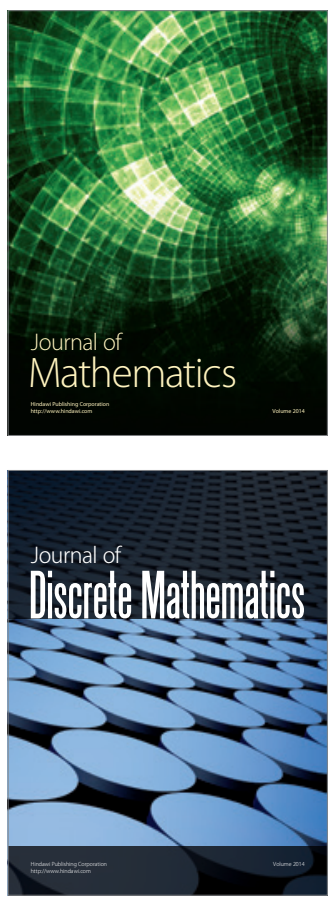

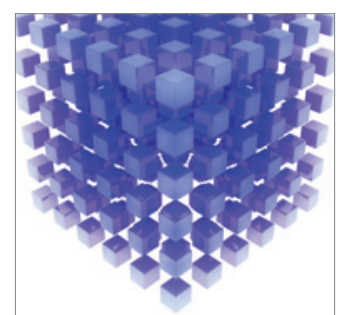

Mathematical Problems in Engineering
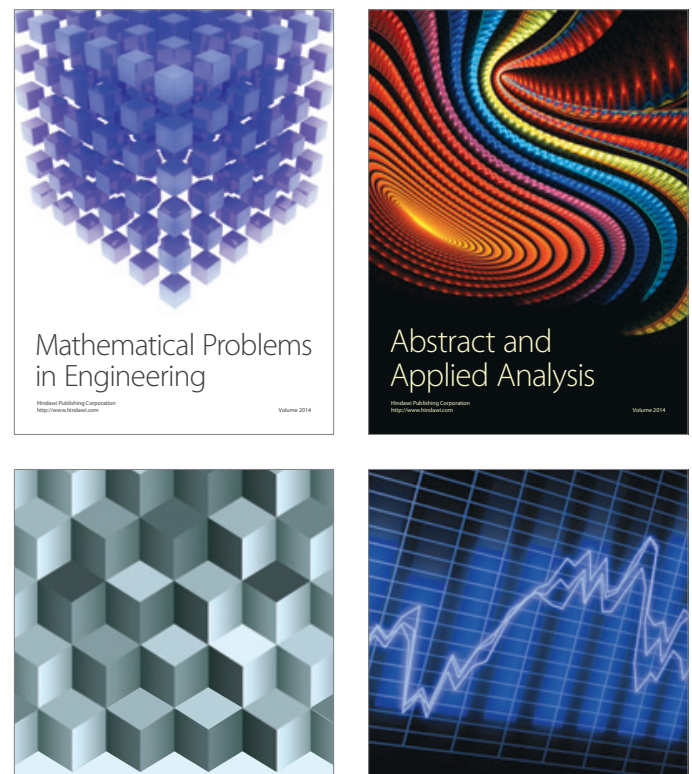

Journal of

Function Spaces

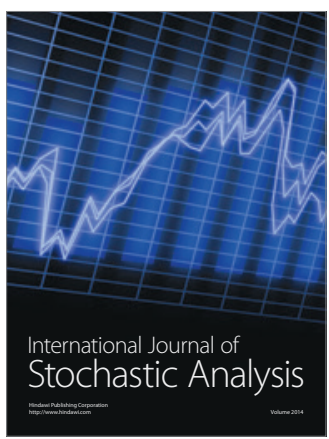

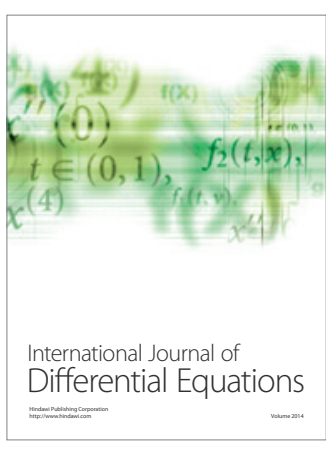
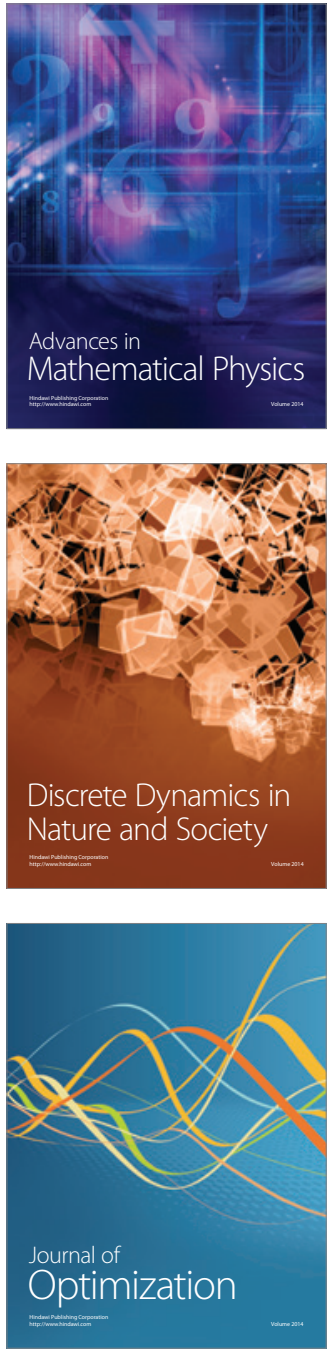